\title{
miR-922 regulates CYLD expression and promotes the cell proliferation of human hepatocellular carcinoma
}

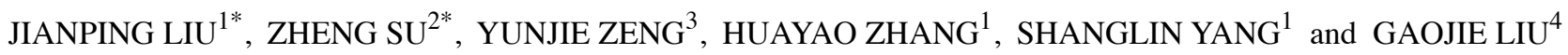 \\ ${ }^{1}$ Department of Hepato-Biliary-Pancreatic Surgery, ${ }^{2}$ Comprehensive Department, and Departments of ${ }^{3}$ Pathology, \\ and ${ }^{4}$ Gastrointestinal Surgery, Sun Yat-sen Memorial Hospital, Sun Yat-sen University, \\ Guangzhou, Guangdong 510120, P.R. China
}

Received May 24, 2016; Accepted June 30, 2016

DOI: $10.3892 /$ or.2017.5431

\begin{abstract}
Evidence reveals that microRNAs (miRNAs) play essential roles in hepatocellular carcinoma (HCC) tumorigenesis. In the present study, we identified an essential role for miR-922 in the development of HCC. We found that miR-922 was significantly upregulated in HCC cells and clinical tissues. Gain and loss of function studies indicated that miR-922 significantly promoted HCC cell proliferation. We subsequently identified that cylindromatosis (CYLD) was a target gene of miR-922. Moreover, miR-922 decreased CYLD expression, subsequently upregulating the expression of c-Myc and cyclin D1, while downregulating p-Rb expression. Furthermore, knockdown of CYLD expression by siRNA partially counteracted the tumor suppressive effect of the inhibitor of miR-922, miR-922-in. Taken together, our findings indicate that miR-922 plays a key role in the promotion of HCC cell proliferation, and strongly suggest that exogenous miR-922 may have therapeutic value for treating HCC.
\end{abstract}

\section{Introduction}

Hepatocellular carcinoma (HCC) is the fifth most common cancer worldwide and the third most common cause of cancer-related mortality (1). Despite improvements in surgical techniques and other treatments for $\mathrm{HCC}$, the prognosis of HCC patients remains unsatisfactory. It is imperative to understand the molecular mechanisms of HCC and find optimized strategies to improve HCC treatment (2).

A growing number of studies have revealed that microRNAs (miRNAs) are highly conserved non-coding

Correspondence to: Dr Jianping Liu, Department of HepatoBiliary-Pancreatic Surgery, Sun Yat-sen Memorial Hospital, Sun Yat-sen University, 107 Yan Jiang West Road, Guangzhou, Guangdong 510120, P.R. China

E-mail: jianpingliu2011@163.com

"Contributed equally

Key words: miR-922, human hepatocellular carcinoma, CYLD, cell proliferation
RNA oligonucleotides (18-25 nt long) that play essential roles in regulating diverse biological processes, such as cell proliferation, invasion, migration, apoptosis and the cell cycle (3-5). miRNAs have been indicated in the regulation of cancer development. Wang et al revealed that downregulation of miR-122 promoted proliferation, migration and invasion of $\mathrm{HCC}$ by activating epithelial-mesenchymal transition (6). miR-383 was reported to inhibit HCC cell proliferation via targeting a proliferation-inducing ligand (APRIL) (7). Furthermore, Zhang et al indicated that miR-381 inhibited cell growth and invasion by targeting the liver receptor homolog-1 in HCC (8). However, the biological function of miR-922 and the related mechanisms in HCC remain unclear.

Cylindromatosis (CYLD), a deubiquitination enzyme, is a tumor-suppressor gene in different types of cancer $(9,10)$. Herein, we indicated that expression of miR-922 was upregulated in HCC cells and clinical tissues. Overexpression of miR-922 promoted HCC cell proliferation. Bioinformatic analyses revealed that miR-922 is able to bind to the 3' untranslated region (3'UTR) of CYLD mRNA to prevent its translation, which was confirmed by luciferase reporter assay. Further experiments indicated that CYLD downregulation counteracted suppress of HCC cell proliferation by the inhibitor of miR-922, miR-922-in. Together, our data suggest that miR-922 overexpression promotes HCC cell growth through the regulation of CYLD expression.

\section{Materials and methods}

Cell culture. Human HCC cell lines Huh7, MHCC97H, HepG2, QGY-7703, Hep3B, MHCC97L, HCCC-9810 and BEL-7402 were obtained from the American Type Culture Collection (ATCC; Manassas, VA, USA), and were cultured in Dulbecco's modified Eagle's medium (Gibco, Grand Island, NY, USA) supplemented with $10 \%$ fetal bovine serum (FBS; Sigma, St. Louis, MO, USA), and $100 \mathrm{U} / \mathrm{ml}$ of penicillin-streptomycin (Invitrogen, Carlsbad, CA, USA). A human hepatic cell line THLE3 was purchased from the China Center for Type Culture Collection (CCTCC; Wuhan, China), and was cultured in McCoy's 5A modified medium (Invitrogen, Life Technologies, Carlsbad, CA, USA) supplemented with $10 \%$ FBS and $100 \mathrm{U} / \mathrm{ml}$ of penicillin-streptomycin. All cells were maintained at $37^{\circ} \mathrm{C}$ in a humidified incubator containing $5 \% \mathrm{CO}_{2}$. 
Clinical specimens. Eight pairs of primary human HCC tissue samples and adjacent normal tissue (ANT) samples were obtained from HCC patients at the Department of Hepato-Biliary-Pancreatic Surgery, Sun Yat-sen Memorial Hospital, Sun Yat-sen University (Guangzhou, China), following surgical dissections. Written informed consent was obtained from each participant. The present study was approved by the Ethics Committee of Sun Yat-sen Memorial Hospital, Sun Yat-sen University. Tissues were snap-frozen in liquid nitrogen.

Plasmids, small interfering RNA and transfection. miR-922 mimic, miR-922 inhibitor (miR-922-in) and negative controls were purchased from GeneCopoeia Co., Ltd. (Guangzhou, China) and transfected into OS cells using Lipofectamine 2000 reagent (Invitrogen) according to the manufacturer's protocol. The CYLD ORF was amplified from the Hep3B cell cDNA and subcloned into pEGFP-N3 (Invitrogen). The wild-type 3'UTR of CYLD was synthesized and subcloned into the firefly luciferase reporter (RiboBio, Guangzhou, China), and then transfected into HCC cells using Lipofectamine 2000 reagent (Invitrogen) according to the manufacturer's instructions. shRNA lentiviruses against CYLD were purchased from GeneCopoeia (Co., Ltd.) and transfected into Hep3B cells in 24-well plates using Lipofectamine 2000 reagent (Invitrogen) according to the manufacturer's instructions.

RNA extraction and real-time quantitative PCR. Total RNA was extracted using TRIzol reagent (Invitrogen), and then cDNA was synthesized from $5 \mathrm{ng}$ of total RNA using the miRNA First-Strand cDNA Synthesis SuperMix (TransGen Biotech, Beijing, China). The expression of miR-922 was detected by TransScript ${ }^{\circledR}$ Green miRNA Two-Step qRT-PCR SuperMix (TransGen Biotech). In addition, the relative miR-922 expression levels after normalization to U6 were calculated. The cyclin D1 and MYC levels were quantified using qRT-PCR with the TaqMan ${ }^{\circledR}$. We assessed the RNA expression according to relative quantification using the $2^{-\Delta \Delta C t}$ method to determine the fold-change in the expression. Each sample was analyzed in triplicate and the mean expression level was calculated.

MTT and colony formation assays. For the MTT assay, at four different time points $(24,48,72$ and $96 \mathrm{~h}) 20 \mu \mathrm{l}$ of $5 \mathrm{mg} / \mathrm{ml}$ MTT solution (Sigma-Aldrich, St. Louis, MO, USA) was added to each well, followed by incubation for $4 \mathrm{~h}$ at $37^{\circ} \mathrm{C}$ and the absorbance was read at $490 \mathrm{~nm}$ by a Thermo Scientific Multiskan (Thermo Fisher Scientific, USA). For the colony formation assays, Hep3B cells after transfection were harvested and seeded into 6 -well plates at $1 \times 10^{3}$ cells/well. Two weeks after seeding, the cells were stained with $0.1 \%$ crystal violet for $1 \mathrm{~min}$ and fixation with $10 \%$ formaldehyde for $10 \mathrm{~min}$. Images were captured and the colonies were counted. Each time point was repeated in three wells and the experiment was independently performed for three times.

Anchorage-independent growth assay. One thousand HepG2 cells were trypsinized and resuspended in $2 \mathrm{ml}$ complete medium plus $0.3 \%$ agar (Sigma). The agar-cell mixture was plated on top of a bottom layer comprising $0.66 \%$ complete medium agar mixture. The plates were incubated at $37^{\circ} \mathrm{C}$ for $>2$ weeks until the visible colonies had formed, and colonies $>0.1 \mathrm{~mm}$ in diameter were counted. The experiment was performed independently three times for each cell line.

Luciferase assays. Cells were transferred with miR-922, or miR-922-in, or miR-922-mut into 24-well plates at a density of 30,000 cells/well. After $24 \mathrm{~h}$, the cells were co-transfected with the pRL-TK plasmid (Promega, Madison, WI, USA) containing the Renilla luciferase gene, and various constructs containing the seed sequence of CYLD 3'UTR. Luciferase reporter assays were performed using the Dual-Luciferase reporter assay system according to the manufacturer's instructions.

Western blotting. The cultured cells were washed with $1 \mathrm{X}$ phosphate-buffered saline (PBS) and lysed with RIPA buffer. Equal quantities of protein $(30 \mu \mathrm{g})$ were used for the detection of CYLD, c-Myc, cyclin D1, Rb and p-Rb protein $(1: 1,000)$ and $\alpha$-tubulin $(1: 1,000)$ (both from Cell Signaling Technology, Danvers, MA, USA) was used as the loading control. In addition, the membranes were washed with PBS and incubated with the respective goat anti-rabbit secondary antibodies (Beyotime Biotechnology, Shanghai, China). The bound antibodies were detected using an ECL kit. Quantity One software was used to quantify protein band intensities.

Statistical analysis. All results in the present study were statistically presented as mean \pm standard error of the mean using SPSS 19.0 (IBM). P<0.05 was considered to indicate a statistically significant result by Student's t-test.

\section{Results}

miR-922 expression is upregulated in HCC cell lines and HCC tissues. To detect the expression of miR-922 in HCC cells, qRT-PCR was used to analyze miR-922 expression in HCC cells and human hepatic cell line THLE3. Expression of miR-922 was markedly upregulated in the HCC cell lines compared with that in the THLE3 cells (Fig. 1A). We also evaluated the expression of miR-922 in HCC specimens. As showed in Fig. 1B, upregulation of miR-922 was observed in the HCC tissues compared with adjacent non-tumor tissues (ANT). Collectively, miR-992 may play a tumor-promoting role in the progression of HCC.

miR-922 promotes, while its inhibitor miR-922-in suppresses $H C C$ cell proliferation. To investigate the biological function of miR-922 in HCC, Hep3B cells were transfected with miR-922 mimic, miR-922-in or negative controls. Transfection efficiency was confirmed by qRT-PCR (Figs. 2A and 3A). Cell growth assay showed that upregulation of miR-922 efficiently promoted the proliferation of Hep3B cells (Fig. 2B). Conversely, a significant decrease in the number of Hep3B cells was observed in the miR-922-in group (Fig. 3B). Notably, colony formation assays indicated that miR-922 significantly promoted, while miR-922-in markedly inhibited the ability of colony formation in the Hep3B cells (Figs. 2C and 3C). Results of anchorage-independent growth showed that miR-922-transfected Hep3B cells formed more and larger colonies (Fig. 2D). 
A

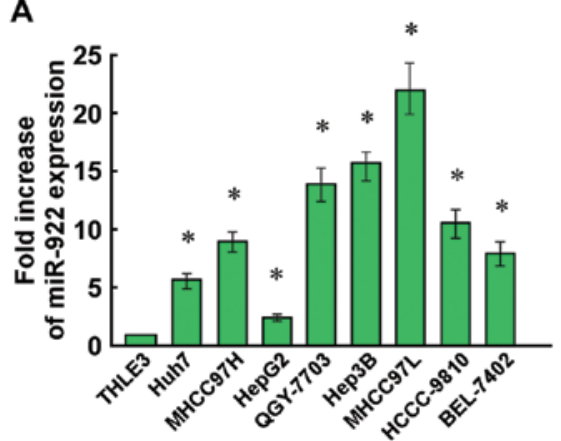

B

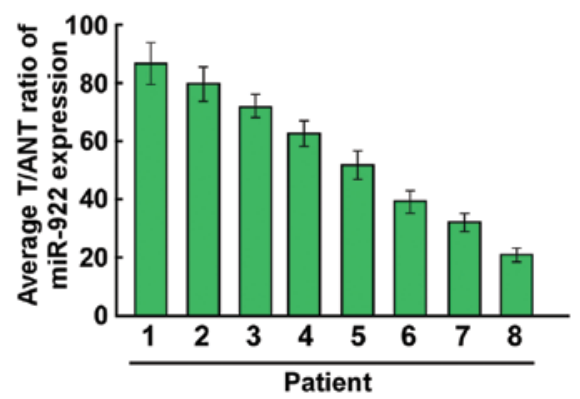

Figure 1. Expression of miR-922 in human hepatocellular carcinoma (HCC) cell lines and tissues. (A) Real-time PCR analysis of miR-922 expression in the human hepatic cell line THLE3 and HCC cell lines, including Huh7, MHCC97H, HepG2, QGY-7703, Hep3B, MHCC97L, HCCC-9810 and BEL-7402. (B) Relative miR-922 mRNA expression levels in 8 paired primary HCC tissues (T) and the matched adjacent normal tissues (ANT) from the same patients were detected by PCR analysis, and the T/ANT ratios are shown. Experiments were repeated at least three times. Each bar represents the mean of three independent experiments; ${ }^{\mathrm{P}}<0.05$.

A

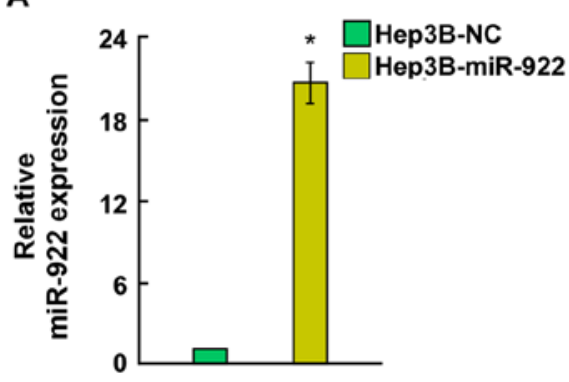

B

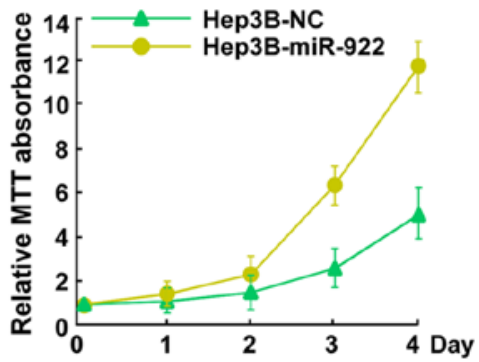

C

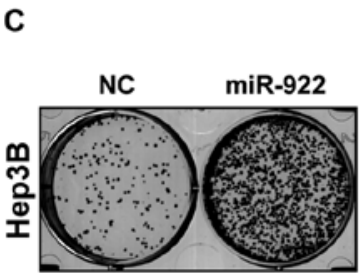

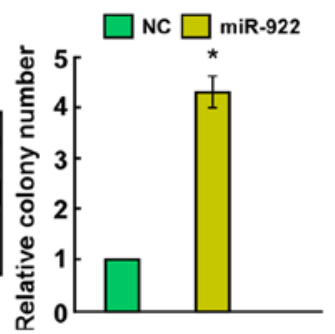
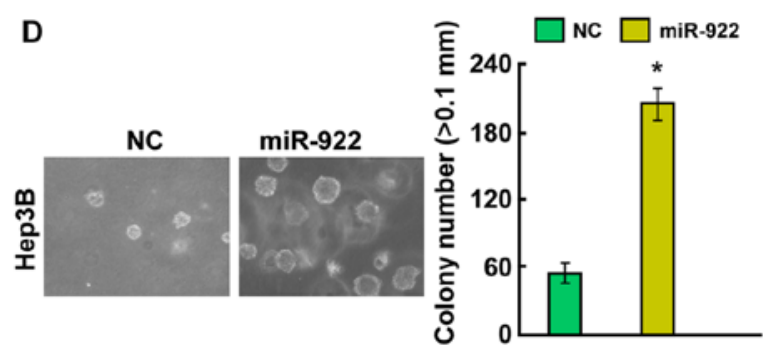

Figure 2. Upregulation of miR-922 promotes HCC cell proliferation. (A) Validation of miR-922 expression levels after transfection of miR-922 mimic by PCR analysis. (B) MTT assays revealed that miR-922 increased the growth of Hep3B cells. (C) Representative micrographs (left) and quantification (right) of crystal violet-stained cell colonies. (D) Upreguation of miR-922 promoted the anchorage-independent growth of Hep3B cells. Representative micrographs (left) and quantification of colonies that were $>0.1 \mathrm{~mm}$ (right). Each bar represents the mean of three independent experiments; " $\mathrm{P}<0.05$.

In contrast, Hep3B cells after transfection with miR-922-in showed a significant reduction in the ability to form colonies on soft agar (Fig. 3D). Collectively, miR-922 promoted the proliferation of HCC cells in vitro.

miR-922 directly targets CYLD by binding to its 3'UTR and alters levels of proteins related to cell proliferation in Hep3B cells. In order to confirm whether CYLD is the direct target of miR-4262, we identified CYLD as a potential target of miR-922 using bioinformatic analysis (TargetScan). We found that the 3'UTR of CYLD was complementary to the miR-922 seed region (Fig. 4A). Hep3B cells were transfected with miR-922 mimic, miR-922-in or negative controls, and the effect of miR-922 or miR-922-in on the expression of CYLD was detected by western blotting. The results indicated that CYLD protein expression was significantly decreased in the Hep3B cells stably expressing miR-922, whereas CYLD protein expression was markedly increased by miR-922-in (Fig. 4B). In addition, we tested whether miR-922 directly targets CYLD by luciferase reporter assay. The results showed that overexpression of miR-922 markedly reduced the luciferase activity of the CYLD wild-type 3'UTR, whereas miR-922-in increased this activity, and the luciferase activity of the CYLD wild-type 3'UTR was not affected by the miR922-mut (Fig. 4C), indicating that miR-922 can bind to the CYLD 3'UTR. Collectively, these results demonstrated that CYLD is a direct target of miR-922.

CYLD was reported to negatively regulate $\mathrm{NF}-\kappa \mathrm{B}$ activity, and Jun $\mathrm{N}$-terminal kinase and $\mathrm{Wnt} / \beta$-catenin signaling pathways (11-13). In the present study, we evaluated the mRNA of the CYLD downstream genes (cyclin D1, c-Myc, Rb and p-Rb). Results of quantitative PCR revealed a significant increase in the mRNA levels of cyclin D1 and c-Myc, while miR-922-in showed the opposite effect in the Hep3B cells (Fig. 4D). 

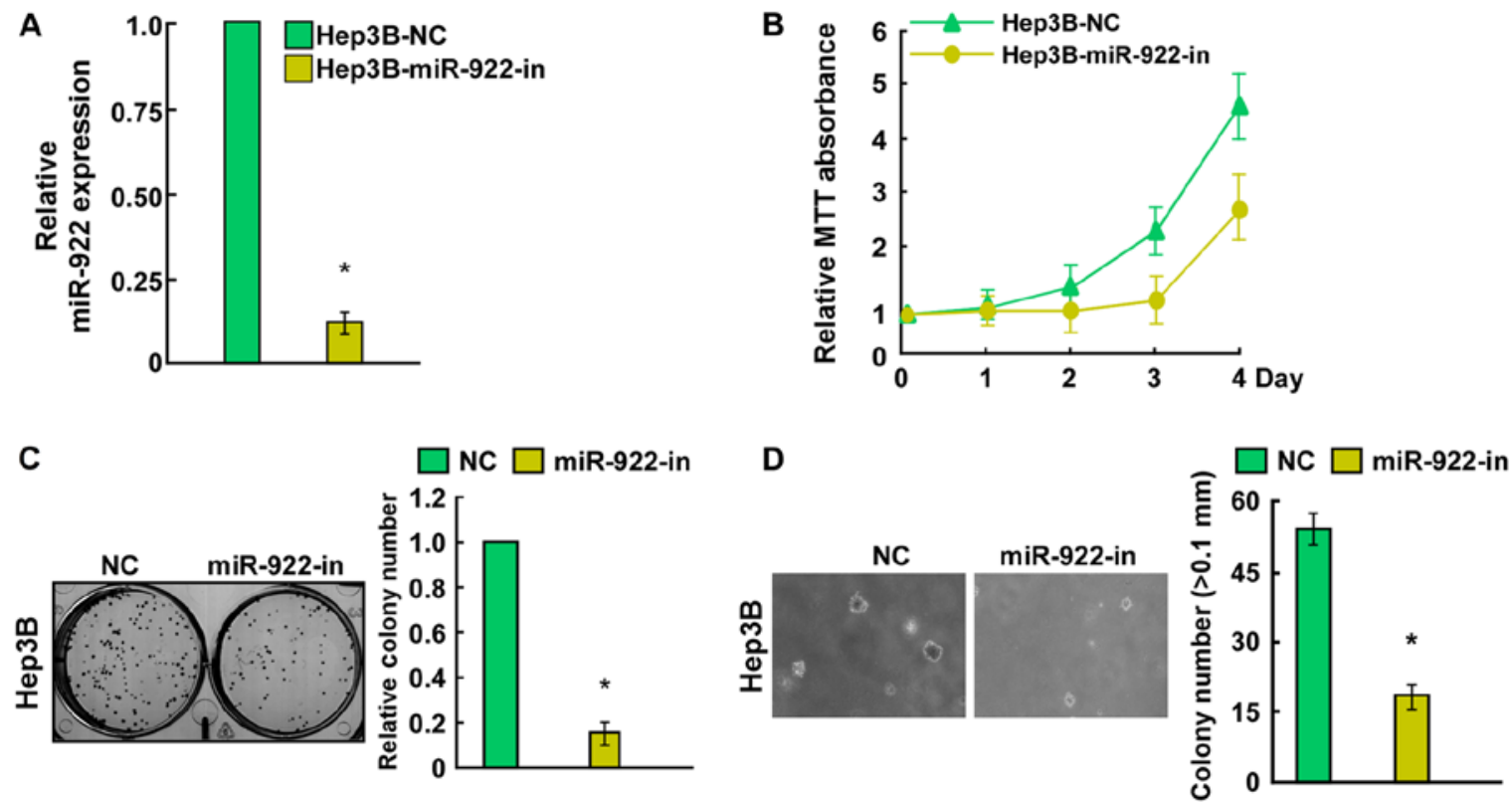

Figure 3. Inhibition of miR-922 suppresses HCC cell proliferation. (A) Validation of miR-922 expression levels after transfection of its inhibitor miR-922-in by PCR analysis. (B) MTT assays revealed that miR-922-in suppressed the growth of Hep3B cells. (C) Representative micrographs (left) and quantification (right) of crystal violet-stained cell colonies. (D) miR-922-in inhibited the anchorage-independent growth of Hep3B cells. Representative micrographs (left) and quantification of colonies that were $>0.1 \mathrm{~mm}$ (right). Each bar represents the mean of three independent experiments; ${ }^{*} \mathrm{P}<0.05$.

A

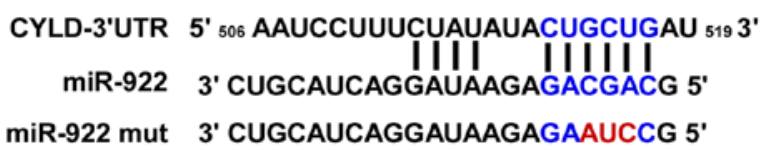

C

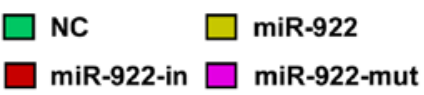

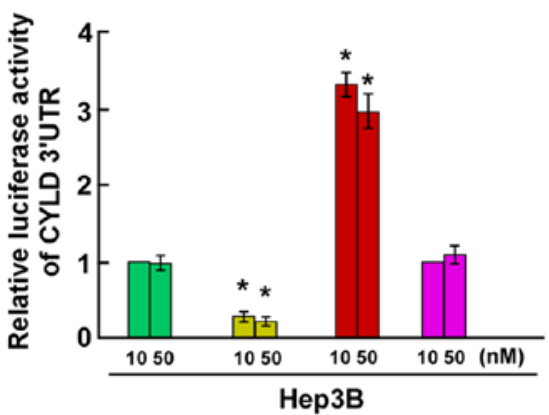

E

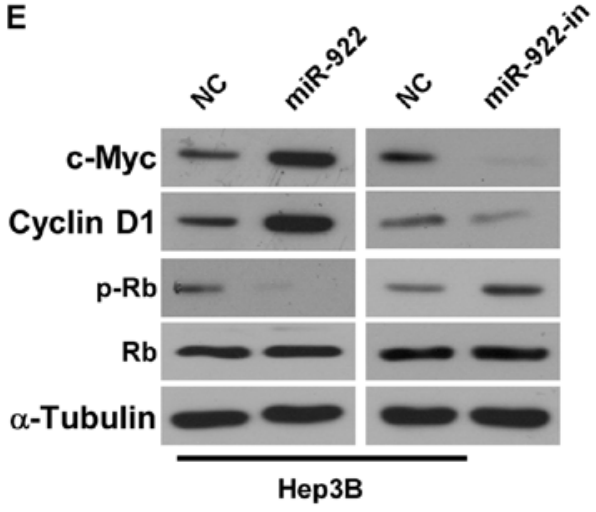

B

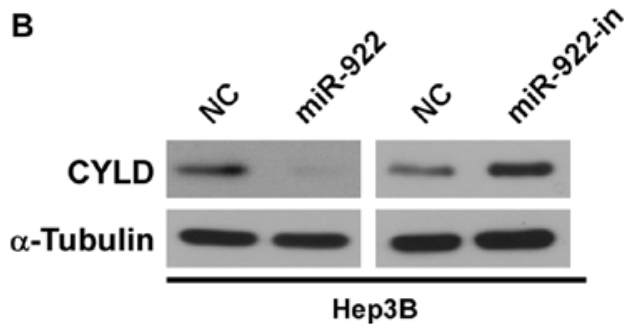

D
NC
$\square$ miR-922
$\square$ miR-922-in $\square$ miR-922-mut

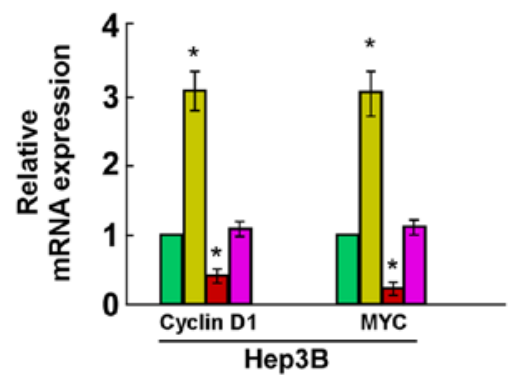

Figure 4. miR-922 suppresses CYLD expression by directly targeting the CYLD 3'UTR and alters the levels of proteins related to proliferation in Hep3B cells. (A) Predicted miR-922 target sequence in the 3'UTR of CYLD (CYLD-3'UTR) and the positions of three mutated nucleotides (red) in the 3'UTR of miR-922-mut. (B) CYLD protein expression in Hep3B cells transfected with miR-922 or miR-922-in was detected by western blot analysis. $\alpha$-tubulin served as the loading control. (C) Luciferase reporter assay of Hep3B cells transfected with the pGL3-CYLD-3'UTR reporter and miR-922 or miR-922-in or miR-922-mut with increasing amounts $(10$ and $50 \mathrm{nM})$ of oligonucleotides. (D) Real-time PCR analysis of the expression of cyclin D1 and MYC in Hep3B cells. (E) Western blot analysis of protein expression of cyclin D1, C-Myc, p-Rb and Rb in the different Hep3B cells. $\alpha$-tubulin was used to serve as the loading control; ${ }^{*} \mathrm{P}<0.05$. 
A

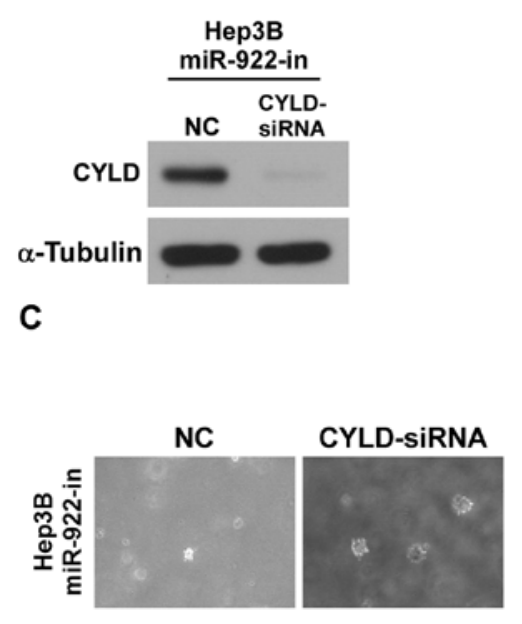

B

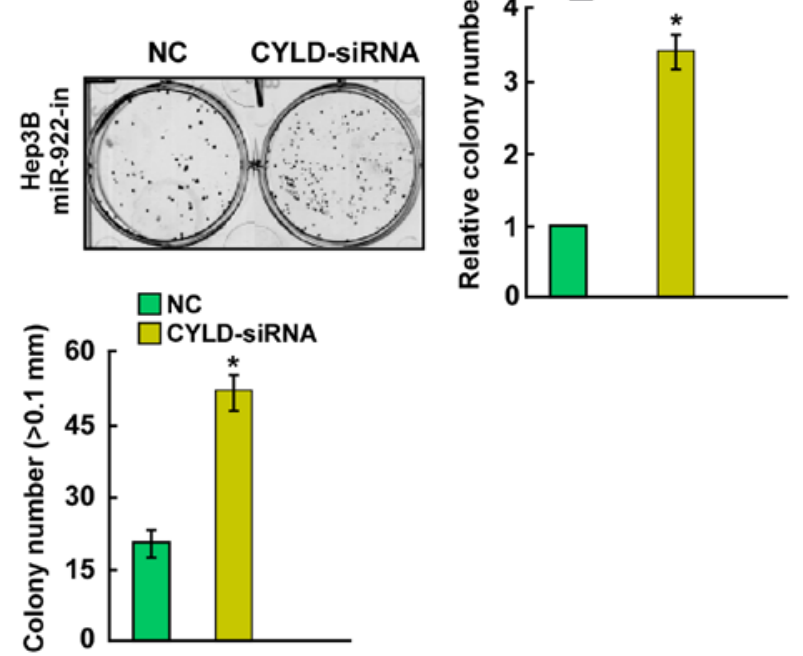

Figure 5. CYLD downregulation counteracts the cell proliferation arrest by miR-922-in. (A) Western blot analysis verified that silencing of CYLD effectively decreased the expression of CYLD in the miR-922-in-transfected Hep3B cells. (B) miR-922-in-transfected Hep3B cells after transfection with CYLD-siRNA increased cell colony formation. (C) miR-922-in-transfected Hep3B cells after transfection with CYLD-siRNA had increased anchorage-independent growth. Representative micrographs (left) and quantification of colonies that were $>0.1 \mathrm{~mm}$ (right). Each bar represents the mean of three independent experiments; ${ }^{*} \mathrm{P}<0.05$.

Western blot analysis showed that the levels of cyclin D1 and $\mathrm{c}-\mathrm{Myc}$ were increased while the level of $\mathrm{p}-\mathrm{Rb}$ was decreased in the miR-922 group compared with the miR-NC group, while miR-922-in showed the opposite function (Fig. 4E). Taken together, our results demonstrated that miR-922 functionally modulated CYLD, and then regulated cellular proliferation regulators, cyclin D1, c-Myc and p-Rb, thus relevant to cell proliferation. These results demonstrated that miR-922 could combine with CYLD 3'UTR and play a role in suppressing the expression of the CYLD gene.

CYLD downregulation counteracts the cell proliferation arrest by miR-922-in. CYLD protein expression was suppressed in the miR-922-in transfected Hep3B cells using CYLD-siRNA (Fig. 5A). Knockdown of CYLD enhanced cell colony formation and anchorage-independent growth ability of miR-922-in-transfected Hep3B cells (Fig. 5B and C). Taken together, these results suggest that the tumor-suppressive effect of miR-922-in is mediated by regulation of CYLD in HCC.

\section{Discussion}

In the present study, we characterized the functional significance of miR-922 in hepatocellular carcinoma (HCC). We demonstrated that miR-922 expression was upregulated in HCC cell lines and clinical tissues. Moreover, ectopic miR-922 expression promoted cell proliferation of the Hep3B cells. Furthermore, miR-922 overexpression increased Hep3B cell colony formation and anchorage-independent growth ability. Additionally, we identified cylindromatosis (CYLD) as a direct target of miR-922. Knockdown of CYLD counteracted the cell proliferation arrest by miR-922-in in the HCC Hep3B cells. These results indicated that miR-922 may function as an oncogene in HCC.
An increasing number of studies indicated that dysregulation of miRNAs plays essential roles in multiple biological processes, such as cell invasion, migration, proliferation, apoptosis, cell cycle and angiogenesis (14-16). miR-765 was found to promote HCC cell proliferation (17). miR-1180 was reported to be significantly increased and to promote the proliferation of HCC (18). Furthermore, miR-379-5p acted as a tumor suppressor and inhibited cell invasion and metastasis in HCC (19). Zhou et al indicated that miR-761 was upregulated and regulated tumorigenesis in HCC (20). However, to date, the biological function and regulatory mechanisms of miR-922 in HCC tumorigenesis have not been elucidated. Currently, we demonstrated that miR-922 acts as a tumor promoter and promotes the cell proliferation of HCC.

The CYLD gene encoding a deubiquitinating enzyme, has been identified as a tumor-suppressor gene and is downregulated in many types of cancer, including HCCs, gliomas, melanoma and colon carcinomas (21-24). CYLD is a direct target of miR-454, the increased expression of which resulted in CYLD reduction and promoted cell proliferation of human colorectal cancer cells (25). miR-501-5p was reported to regulate CYLD expression and promote cell proliferation in human HCC (26). However, whether miR-922 can directly target CYLD in HCC remains unknown. In the present study, the results of the bioinformatic analysis indicated sequence complementarity between miR-922 and the 3'UTR of CYLD and was confirmed by luciferase assays. Further experiment revealed that knockdown of CYLD by siRNA counteracted the cell proliferation arrest by miR-922-in. These results suggest that miR-922 directly regulates CYLD expression in HCC.

In summary, the present study indicated that miR-922 promoted HCC cell proliferation by suppressing CYLD expression and offers a new insight into the mechanisms of miR-922 in HCC, suggesting that miR-922 could be a potential therapeutic target in HCC. 


\section{Acknowledgements}

The present study was supported by the Guangzhou Major Projects on Collaborative Innovation of Industry (1561000198).

\section{References}

1. El-Serag HB and Rudolph KL: Hepatocellular carcinoma: Epidemiology and molecularcarcinogenesis. Gastroenterology 132 2557-2576, 2007.

2. Callegari E, Gramantieri L, Domenicali M, D'Abundo L, Sabbioni S and Negrini M: MicroRNAs in liver cancer: A model for investigating pathogenesis and novel therapeutic approaches. Cell Death Differ 22: 46-57, 2015.

3. Xie W, Qin W, Kang Y, Zhou Z and Qin A: MicroRNA-340 inhibits tumor cell proliferation and induces apoptosis in endometrial carcinoma cell line RL 95-2. Med Sci Monit 22 : 1540-1546, 2016.

4. Zhang M, Zhuang Q and Cui L: MiR-194 inhibits cell proliferation and invasion via repression of RAP2B in bladder cancer. Biomed Pharmacother 80: 268-275, 2016

5. Jin L, Li Y, Liu J, Yang S, Gui Y, Mao X, Nie G and Lai Y: Tumor suppressor miR-149-5p is associated with cellular migration proliferation and apoptosis in renal cell carcinoma. Mol Med Rep 13: 5386-5392, 2016.

6. Wang N, Wang Q, Shen D, Sun X, Cao X and Wu D: Downregulation of microRNA-122 promotes proliferation, migration, and invasion of human hepatocellular carcinoma cells by activating epithelial-mesenchymal transition. Onco Targets Ther 9 : 2035-2047, 2016

7. Chen L, Guan H, Gu C, Cao Y, Shao J and Wang F: miR-383 inhibits hepatocellular carcinoma cell proliferation via targeting APRIL. Tumour Biol 37: 2497-2507, 2016.

8. Zhang Q, Zhao S, Pang X and Chi B: MicroRNA-381 suppresses cell growth and invasion by targeting the liver receptor homolog-1 in hepatocellular carcinoma. Oncol Rep 35: 1831-1840, 2016.

9. Massoumi R: CYLD: A deubiquitination enzyme with multiple roles in cancer. Future Oncol 7: 285-297, 2011.

10. Massoumi R, Chmielarska K, Hennecke K, Pfeifer A and Fässler R: Cyld inhibits tumor cell proliferation by blocking Bcl-3-dependent NF-kappaB signaling. Cell 125: 665-677, 2006.

11. Ni F, Gui Z, Guo Q, Hu Z, Wang X, Chen D and Wang S: Downregulation of miR-362-5p inhibits proliferation, migration and invasion of human breast cancer MCF7 cells. Oncol Lett 11: 1155-1160, 2016.

12. Pannem RR, Dorn C, Ahlqvist K, Bosserhoff AK, Hellerbrand C and Massoumi R: CYLD controls c-MYC expression through the JNK-dependent signaling pathway in hepatocellular carcinoma Carcinogenesis 35: 461-468, 2014

13. Tauriello DV, Haegebarth A, Kuper I, Edelmann MJ, Henraat M, Canninga-van Dijk MR, Kessler BM, Clevers H and Maurice MM: Loss of the tumor suppressor CYLD enhances Wnt/beta-catenin signaling through K63-linked ubiquitination of Dvl. Mol Cell 37: 607-619, 2010
14. Yang G, Zhang X and Shi J: MiR-98 inhibits cell proliferation and invasion of non-small cell carcinoma lung cancer by targeting PAK1. Int J Clin Exp Med 8: 20135-20145, 2015.

15. Lv H, Zhang Z, Wang Y, Li C, Gong W and Wang X: MicroRNA-92a promotes colorectal cancer cell growth and migration by inhibiting KLF4. Oncol Res 23: 283-290, 2016.

16. Shen F, Cai WS, Feng Z, Li JL, Chen JW, Cao J and Xu B: MiR-492 contributes to cell proliferation and cell cycle of human breast cancer cells by suppressing SOX7 expression. Tumour Biol 36: 1913-1921, 2015.

17. Xie BH, He X, Hua RX, Zhang B, Tan GS, Xiong SQ, Liu LS, Chen W, Yang JY, Wang XN, et al: Mir-765 promotes cell proliferation by downregulating INPP4B expression in human hepatocellular carcinoma. Cancer Biomark 16: 405-413, 2016.

18. Zhou X, Zhu HQ, Ma CQ, Li HG, Liu FF, Chang H and Lu J: MiR-1180 promoted the proliferation of hepatocellular carcinoma cells by repressing TNIP2 expression. Biomed Pharmacother 79: 315-320, 2016.

19. Chen JS, Li HS, Huang JQ, Dong SH, Huang ZJ, Yi W, Zhan GF, Feng JT, Sun JC and Huang XH: MicroRNA-379-5p inhibits tumor invasion and metastasis by targeting FAK/AKT signaling in hepatocellular carcinoma. Cancer Lett 375: 73-83, 2016.

20. Zhou X, Zhang L, Zheng B, Yan Y, Zhang Y, Xie H, Zhou L, Zheng S and Wang W: MicroRNA-761 is upregulated in hepatocellular carcinoma and regulates tumorigenesis by targeting Mitofusin-2. Cancer Sci 107: 424-432, 2016.

21. Urbanik T, Köhler BC, Boger RJ, Wörns MA, Heeger S, Otto G, Hövelmeyer N, Galle PR, Schuchmann M, Waisman A, et al: Down-regulation of CYLD as a trigger for NF- $\kappa \mathrm{B}$ activation and a mechanism of apoptotic resistance in hepatocellular carcinoma cells. Int J Oncol 38: 121-131, 2011.

22. Guo J, Shinriki S, Su Y, Nakamura T, Hayashi M, Tsuda Y, Murakami Y, Tasaki M, Hide T, Takezaki T, et al: Hypoxia suppresses cylindromatosis (CYLD) expression to promote inflammation in glioblastoma: Possible link to acquired resistance to anti-VEGF therapy. Oncotarget 5: 6353-6364, 2014.

23. Ke H, Augustine CK, Gandham VD, Jin JY, Tyler DS, Akiyama SK, Hall RP and Zhang JY: CYLD inhibits melanoma growth and progression through suppression of the JNK/AP-1 and $\beta 1$-integrin signaling pathways. J Invest Dermatol 133: 221-229, 2013.

24. Hellerbrand C, Bumes E, Bataille F, Dietmaier W, Massoumi R and Bosserhoff AK: Reduced expression of CYLD in human colon and hepatocellular carcinomas. Carcinogenesis 28: 21-27, 2007.

25. Liang HL, Hu AP, Li SL, Xie JP, Ma QZ and Liu JY: MiR-454 prompts cell proliferation of human colorectal cancer cells by repressing CYLD expression. Asian Pac J Cancer Prev 16: 2397-2402, 2015

26. Huang DH, Wang GY, Zhang JW, Li Y, Zeng XC and Jiang N: MiR-501-5p regulates CYLD expression and promotes cell proliferation in human hepatocellular carcinoma. Jpn J Clin Oncol 45: 738-744, 2015. 